Ann. Génét. Sél. anim., I969, 1 (2), Io9-II7.

\title{
HYDROCÉPHALIE ET CARDIOPATHIE HÉRÉDITAIRES EN RACE BOVINE LIMOUSINE
}

\author{
J.-J. LAUVERGNE et C. PAVAUX \\ Station centrale de Génétique animale, \\ Centre national de Recherches zootechniques, 78-Jouy-en-Josas \\ Institut national de la Recherche agronomique \\ Laboratoire d'Anatomie, École nationale vétérinaire, 31-Toulouse
}

\section{SOMMAIRE}

Pour expliquer toutes les anomalies apparues dans la descendance du taureau limousin Pompon dès la prime période embryonnaire, nous avons dû dissocier les insuccès de la fécondation (mesurés par les retours à un mois) des anomalies suivantes. Ces dernières, qui consistent en une mortalité très élevée entre 1 mois et 4 mois de gestation et dans la naissance d'un certain nombre d'anormaux hydrocéphales et cardiopathes, seraient causées par un gène dominant porté par Pompon, à pénétrance de $90 \mathrm{p}$. $100 \mathrm{chez}$ les hétérozygotes.

Au centre d'insémination artificielle de Soual (Tarn), un taureau limousin, mis en testage sur la descendance pour la viande en I963, a donné un pourcentage anormalement élevé de retours en chaleurs et des veaux défectueux qui sont tous morts jeunes. La présente étude s'efforce de préciser ces faits et d'en donner une explication génétique.

\section{I. - EXAMEN DES DONNÉES DE TESTAGE}

Pompon $\left(^{1}\right)$ a été mis au testage en même temps que 5 autres taureaux limousins, en mars-avril rg63.

Pour chaque taureau, les inséminations de testage s'échelonnent sur un mois; elles sont faites tous les 6 jours, en 4 fois. On insémine indifféremment toutes

(1) H. B. Limousin, no $8708602 \mathrm{~S}$ o9, né le 24-II-6I chez M. Chedelaud à Puy-I arouse, commune de Linard (Haute-Vienne), abattu le 9-4-64. 
les vaches en chaleur qui se présentent, que ce soit en première, seconde ou troisième intervention. Ensuite, les mâles restent inutilisés jusqu'à ce que l'on connaisse les performances de croissance et de conformation de leurs descendants exploités en veaux de boucherie.

Tous les retours en chaleurs après insémination sont notés et l'on calcule les pourcentages des non-retours dans le mois qui suit (taux de réussite à $30 \mathrm{j}$ ) et dans les 4 mois qui suivent (taux de réussite à $\mathrm{r} 20 \mathrm{j}$ ).

Huit mois après les premières inséminations, on dresse la liste des vaches présumées pleines (celles pour lesquelles l'inséminateur n'a pas été rappelé) et on établit les cartes de déclaration de naissance, qui sont remises aux éleveurs. Ces derniers les renvoient au Centre au fur et à mesure de la naissance des produits.

Un personnel spécialisé du Centre suit alors les veaux ainsi signalé jusqu'à leur abattage à 90 jours, notant la présence d'éventuelles anomalies, l'état de santé, le poids (tous les mois). Lors de l'abattage, outre le poids, on enregistre également le prix à la vente et on donne une note de conformation. Tous les résultats concernant Pompon et ses 5 contemporains sont présentés dans le tableau $\mathrm{I}$.

\section{II. - EXAMEN DU TAUREAU ET DES VEAUX ANORMAUX}

\section{A. - Le taureau}

A part un tic qui le faisait continuellement renacler dans sa mangeoire, le taureau Pompon paraissait parfaitement normal vu de l'extérieur. Son caryotype s'est révélé normal et un électrocardiogramme présentait des variations à peine subnormales. A l'abattage, un examen nécropsique rapide n'a également rien montré d'anormal $\left(^{(1)}\right.$.

\section{B. - Les veaux anormaux}

Io Extérieur:

A la naissance, l'apparence est quasi-normale; progressivement, se développent des signes d'anomalies morphologiques et des troubles fonctionnels : le front se bombe légèrement, les oreilles semblent petites et rejetées en arrière; au cou apparaît une volumineuse " grosseur "; d'importants troubles cardiopulmonaires s'installent : le veau respire difficilement, le moindre effort l'essouffle; certains tremblent sur leur train-arrière, dont les muscles sont peu développés, et dont les articulations sont volumineuses. Ces signes sont d'une importance variable selon les sujets, mais chez tous, ils sont présents (fig. r). Pourvus d'une faible vitalité, ces veaux meurent, sans exception, avant l'âge d'un mois $\left({ }^{2}\right)$.

(1) Ces examens ont été effectués par le $D^{r}$ Vét. M. Dubors, C.R.V.Z., l'Orfrasière 37, Nouzilly, nous le remercions bien vivement.

( ${ }^{2}$ Ces traits ont été rapportés par les premiers observateurs, puis confirmés par le $\mathrm{D}^{\mathrm{r}}$ Vét. G. PERRET, du centre de Soual, qui s'est occupé de réunir les premières données concernant l'anomalie. 


\section{TABLEAU I}

Résultats de testage du taureau limousin Pompon (065) et de ses contemporains au C.I.A. de Soual en 1953

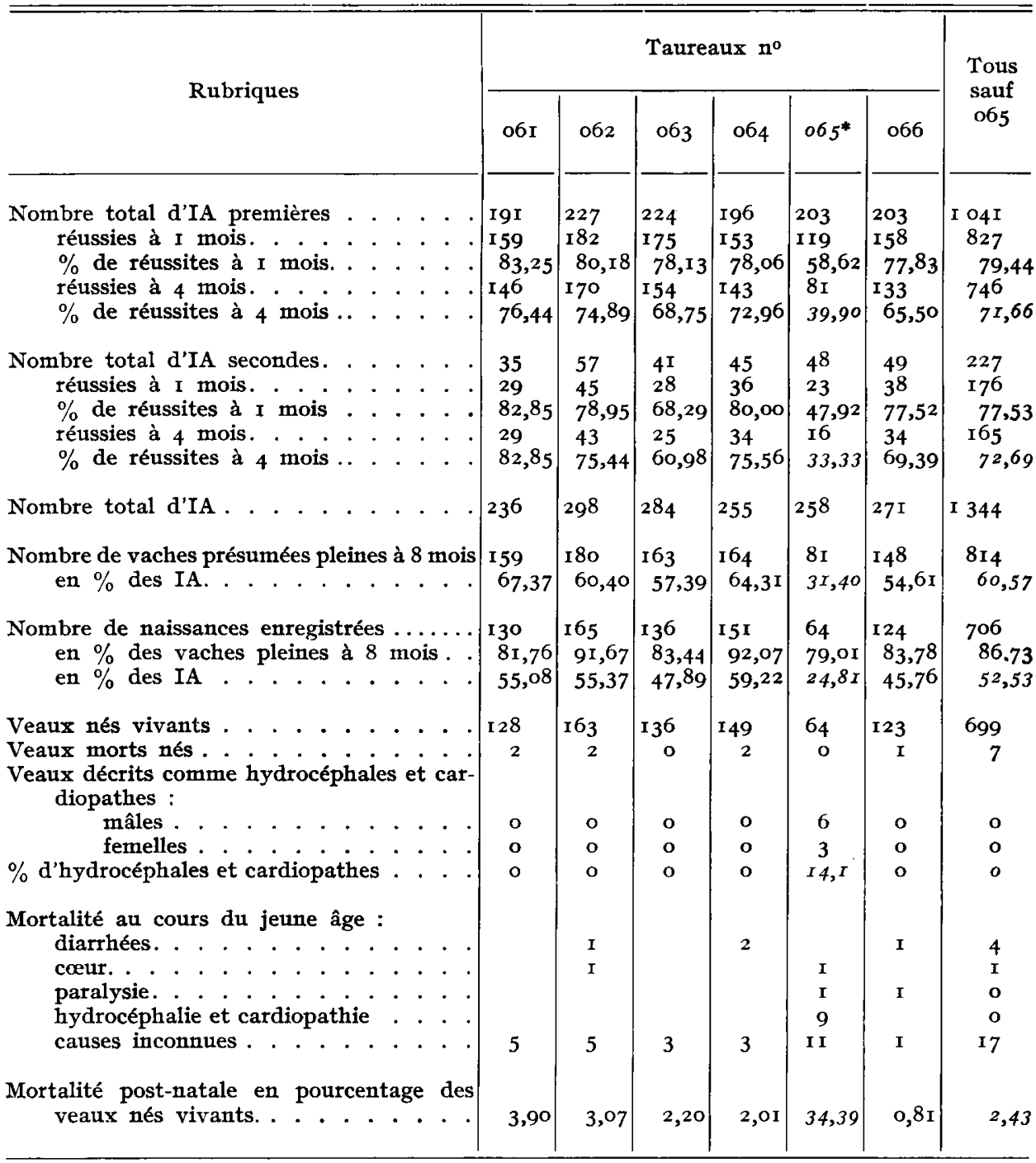

* Pompon. 

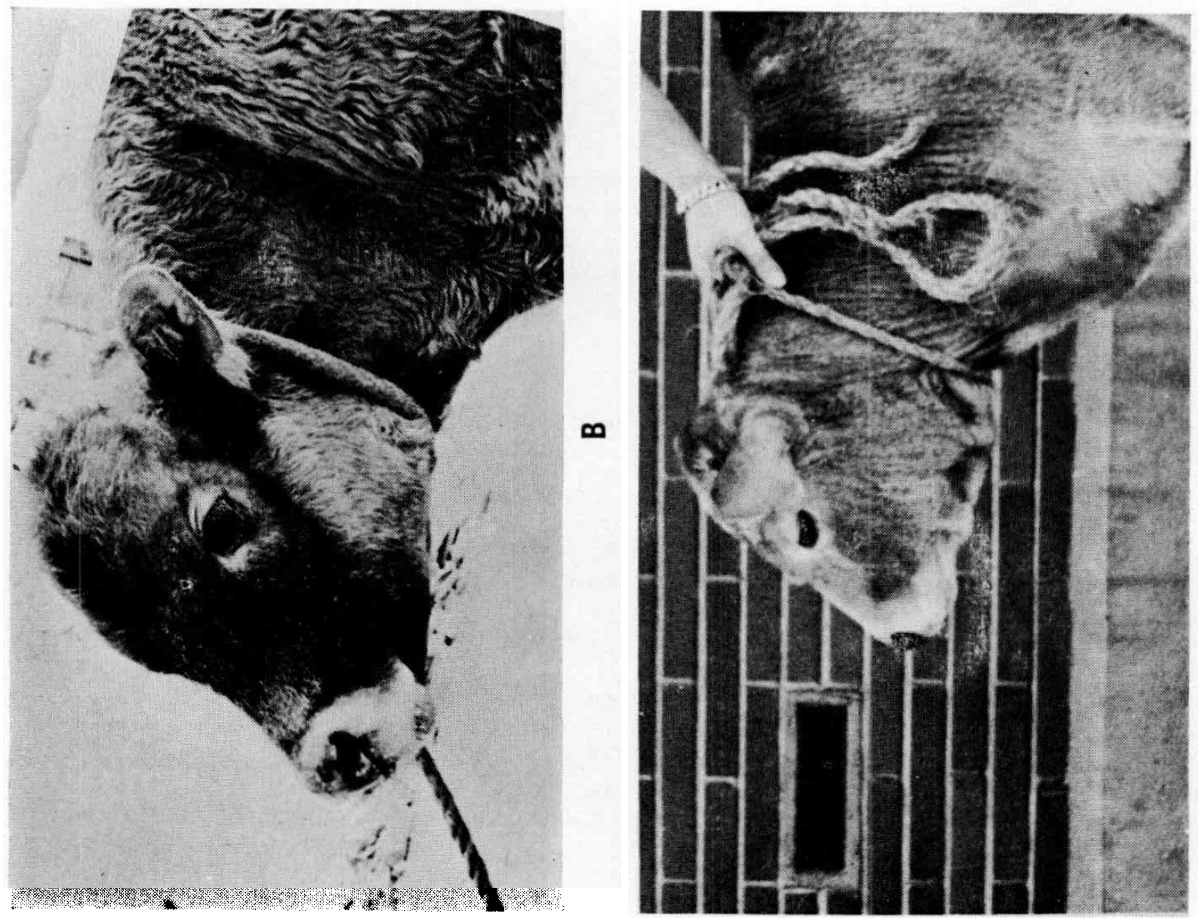

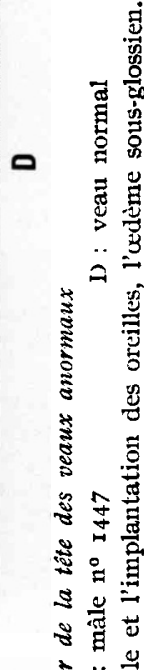
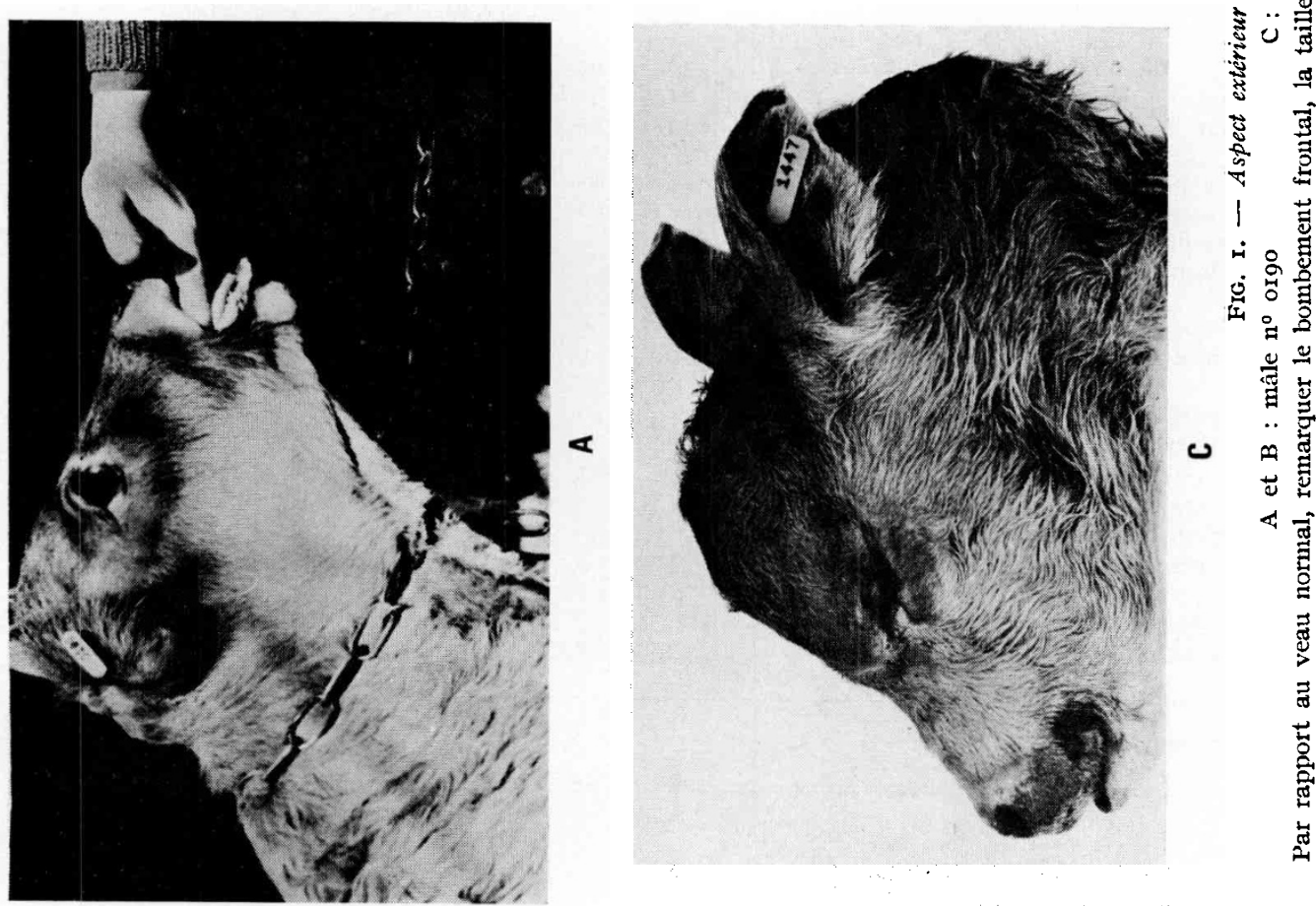

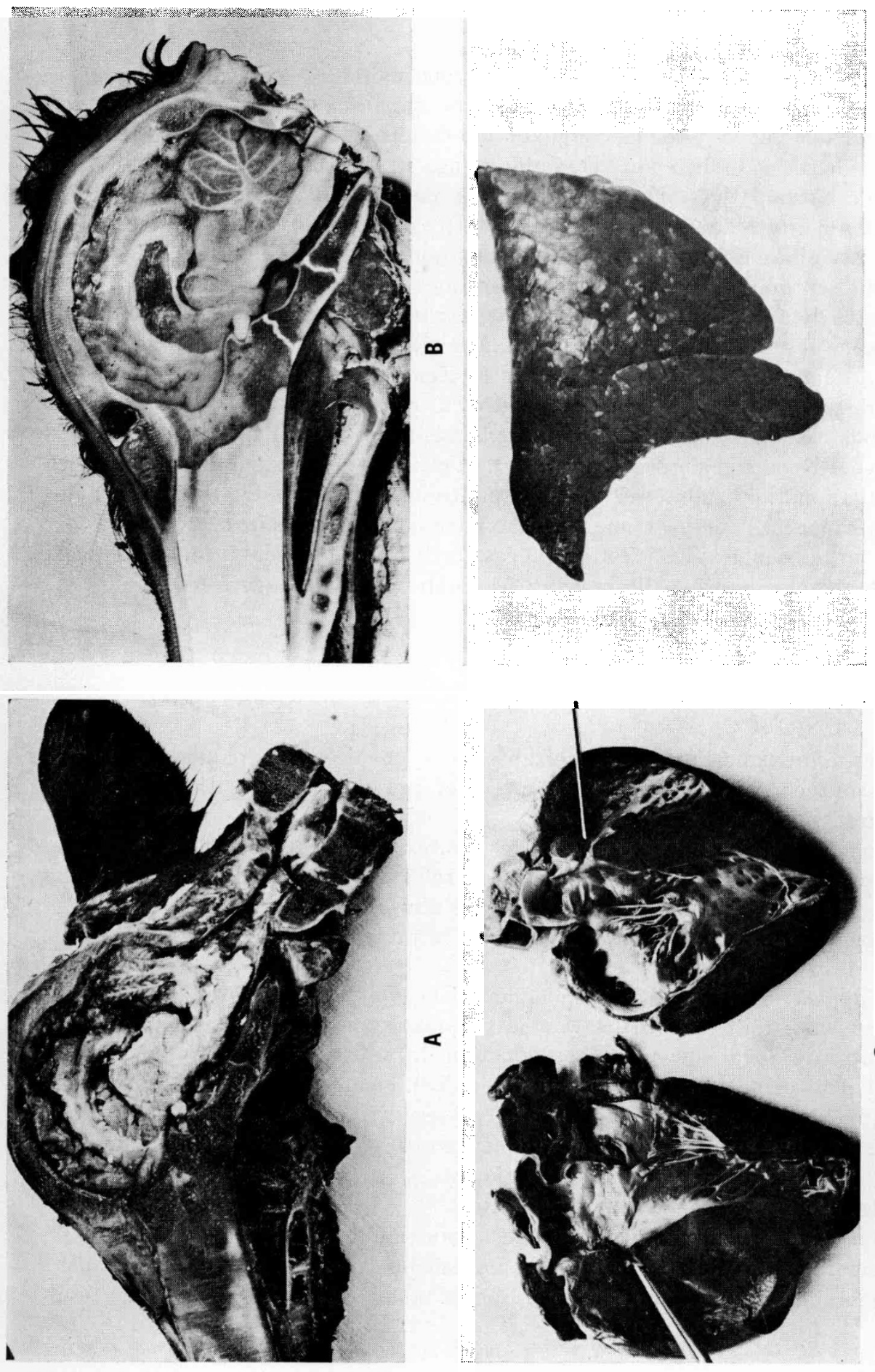

- $M$

㞼

穴寄

官。

岁蒂

온

है..

ธ 4

过

उ

है

\&

흘

今

ง ํㅗㄹ

1 密。

त

施苞

붕

空灵

$\because 8$

范

娄

뭉

ง

诸

สี...

용.

요

호워

论氖苋

13

๓08

प1

४ण 


\section{$2^{\circ}$ Examens nécropsiques:}

Trois veaux ont été adressés à l'École vétérinaire de Toulouse pour autopsie.

Une légère hydrocéphalie ventriculaire apparaît nettement sur une section sagittale de la tête. Les organes interhémisphériques (trigone cérébral et corps calleux) sont distendus et amincis (fig. 2, A et B).

Une cardiopathie constante se traduit par une dilatation des ventricules, surtout du droit. La paroi myocardique est extrêmement flasque. Sur l'un des sujets examinés existait même une communication interventriculaire, tout en haut du septum, sous le muscle pré-aortique (fig. $2, \mathrm{C}$ ).

Cette déficience cardiaque se traduit, sur le plan fonctionnel, par une importante gêne respiratoire : les poumons sont le siège d'une congestion passive alors qu'un œdème important s'installe dans la région sous-glossienne (explication de la " grosseur " au niveau du cou) (fig. I, C et $2, \mathrm{D}$ ).

Tous ces traits sont constants et définissent assez bien l'anomalie héréditaire envisagée. Nous signalerons toutefois que l'examen nécropsique a parfois révélé une inflammation aiguë des voies respiratoires supérieures (glosso-pharyngite, laryngo-trachéite) ainsi qu'une broncho-pneumonie parasitaire $\left({ }^{1}\right)$.

A notre connaissance, c'est le seul cas décrit jusqu'à présent d'un tel complexe morphopathologique héréditaire chez les bovins (LAUVERGNE, I968).

\section{III. - ÉTUDE GÉNÉTIQUE}

Les données concernant la mortalité embryonnaire et la fréquence des anormaux sont consignées dans le tableau I et présentées schématiquement dans la figure 3 .

Comme pour 1'anomalie " tête de mouton " précédemment apparue dans ce même Centre (LAUVERGNE et VISSAC, I963) l'explication génétique la plus simple que nous puissions formuler est celle d'un facteur dominant à pénétrance incomplète porté à l'état hétérozygote par le taureau Pompon et transmis à la moitié de ses descendants.

L'hypothèse de récessivité monofactorielle doit, en effet, être écartée pour deux raisons : tout d'abord les taureaux limousins ne sont utilisés dans la zone du Centre de Soual que pour la production de veaux de boucherie en principe tous abattus vers 1'âge de 3 mois; en second lieu, même si quelques filles d'anciens taureaux limousins porteurs du même gène récessif avaient été élevées et accouplées à Pompon elles n'auraient pu donner une aussi forte proportion d'anormaux (I4,I \%) qui impliquerait une proportion de 56,4\% de femelles hétérozygotes dans les troupeaux de la zone du Centre.

Nous avons indiqué plus haut que le taureau Pompon se distinguait de ses contemporains par des pourcentages anormalement élevés de retour en chaleurs des vaches auxquelles il fut accouplé. Dans le tableau 2, nous présentons ces résul-

(') Nous remercions ici vivement M. le Pr LABIE, de l'École vétérinaire de Toulouse, qui a effectué les examens histologiques. 
tats comparés. Les différences sont hautement significatives pour les pourcentages de retours au cours des 30 premiers jours ainsi que pour les pourcentages cumulés de 30 à I 20 jours. De I20 jours de gestation jusqu'au terme, par contre, la mortalité embryonnaire chez les fœtus engendrés par Pompon n'est pas supérieure à celle des fœtus issus de ses contemporains.

Au total, comparée à celle de ses contemporains, la descendance de Pompon accuse un déficit numérique de $65 \mathrm{p}$. roo (il est né 64 veaux au lieu de I83). En

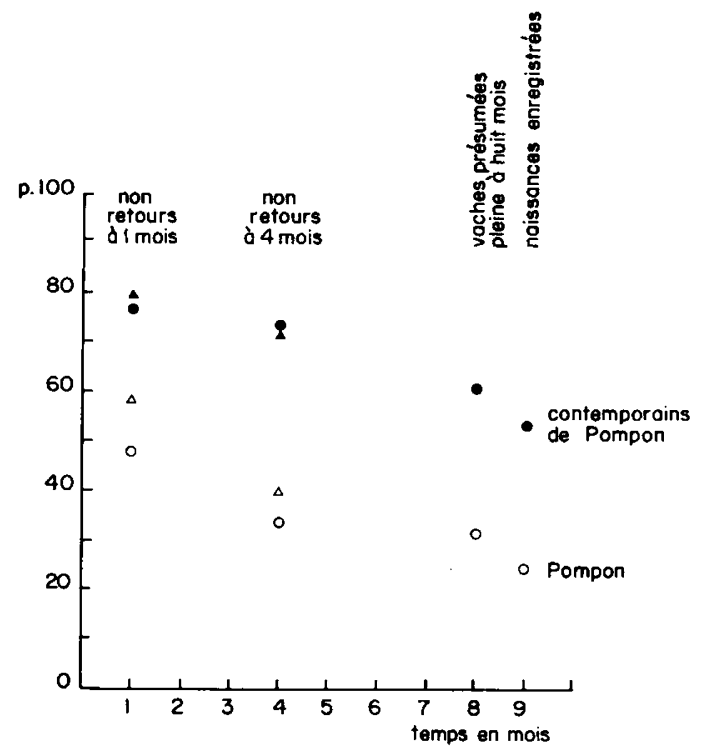

FIG. 3. - Représentation schématique de la chute de fécondité aux différents stades suivant l'insémination avec de la semence de Pompon et de ses contemporains, en pourcentage du nombre des femelles inséminées.

LEGENDE

Non-retours à I mois et à 4 mois seulement
$\Delta$ après IA premières de Pompon.
$O$ après IA secondes de Pompon.
$\Delta$ après IA premières de contemporains de Pompon.
- après IA secondes de contemporains de Pompon.

tenant compte, en outre, des 9 veaux anormaux, c'est un pourcentage de $70 \mathrm{p}$. Ioo de veaux nés porteurs de l'anomalie ou disparus à tous les stades de la vie embryonnaire depuis la fécondation.

Il est impossible d'expliquer cet écart dans le cadre de notre hypothèse puisque, même en admettant une pénétrance de roo p. Ioo, on n'observerait, au plus, que $50 \mathrm{p}$. Ioo de zygotes exprimant une anomalie.

Une explication plus logique dissocierait l'excès des retours à 30 jours de l'action du gène sur les zygotes pour l'attribuer à une mauvaise qualité du sperme du taureau Pompon. Dans ce cas, l'action totale du gène se limiterait à une disparition d'une partie des hétérozygotes entre $I$ mois et 4 mois de gestation, et à la présence d'une anomalie chez un certain nombre d'hétérozygotes survivants qui, hydrocéphales et cardiopathes, sont condamnés à brève échéance. 


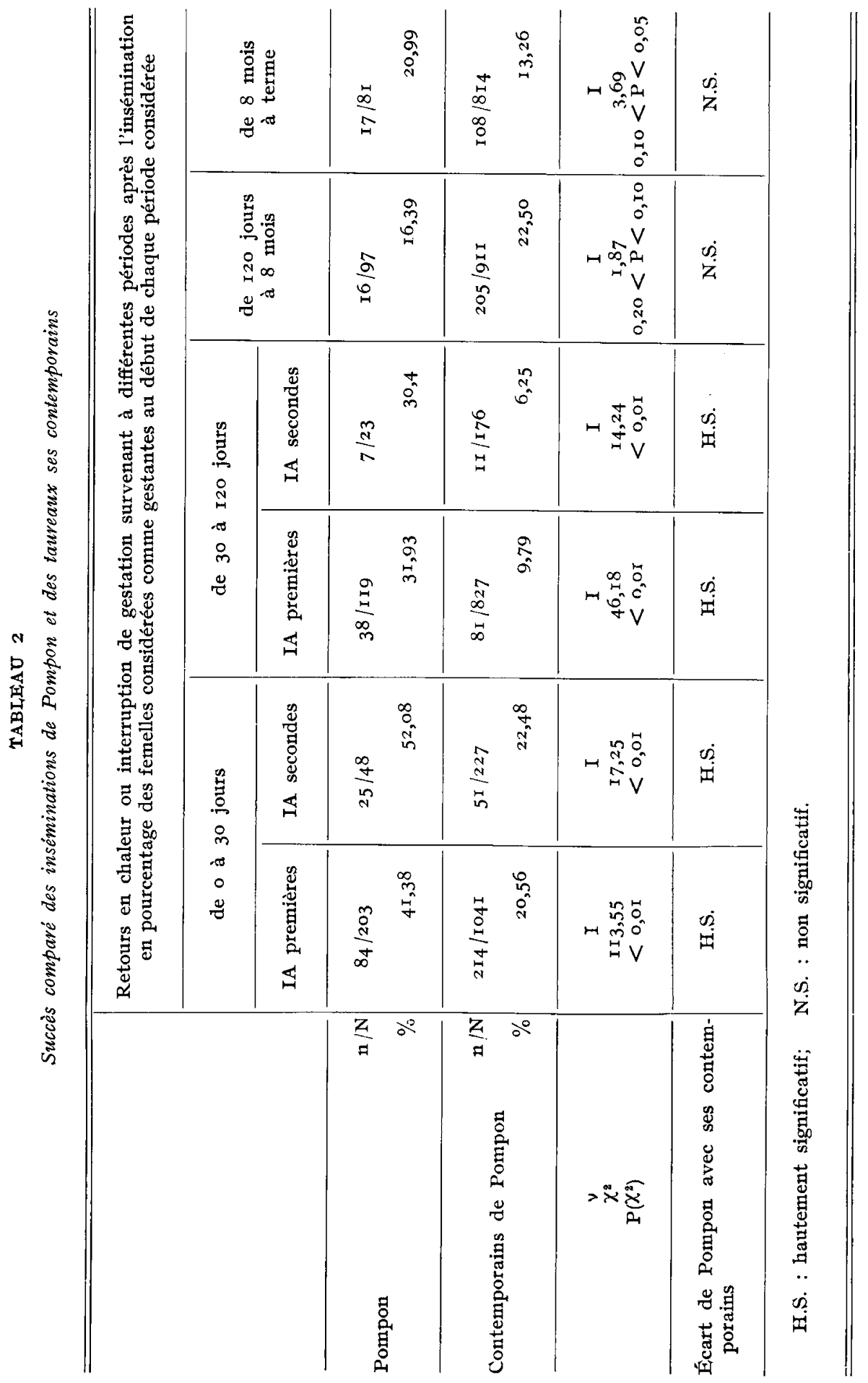


En l'absence du gène d'anomalie, il serait né environ 99 veaux normaux $(99,4)$. On en compte 55 seulement, c'est dire que le gène en question est responsable de la disparition en cours de gestation où consécutivement à des anomalies congénitales décrites d'environ 44 sujets. Ceci correspond à une pénétrance de $0,89 \mathrm{chez}$ les hétérozygotes.

Avec cette interprétation, on aboutit donc à un chiffre acceptable pour la pénétrance. Il reste toutefois à expliquer la mauvaise qualité du sperme de Pompon : est-elle en rapport ou non avec son hétérozygote pour le gène que nous pressentons?

D'une manière plus générale, il faudrait, pour préciser tous ces points, recommencer une expérience de croisement sur un nombre plus grand de femelles. Outre le fait que le taureau a été abattu et que l'on ne dispose que de quelques doses de sperme congelé, on n'envisage guère, vu les pertes essuyées, de mettre en place un nouvel essai.

Reçu pour publication en juin 1968.

\section{SUMMARY}

\section{HEREDITARY HYDROCEPHALUS AND CARDIOPATHY IN THE LIMOUSIN BREED}

Tested at the Soual Artificial Insemination Center (Tarn, France) at the same time as 5 contemporary bulls of the same breed, the Limousin bull Pompon showed marked deviation in the percentages of cows bred to him which subsequently returned into heat. These discrepancies were very significant for the two periods from 2 to 30 days and from 30 to 120 days after insemination. Furthermore, a certain number of the living progeny of both sexes showed the characteristic association of mild hydrocephalus with a serious cardiopathy (cardiac dilation, interventricular communication). The affected individuals were very weak, breathed with great difficulty and died before the age of one month.

In the absence of new data which it would doubtless be difficult to get, in order to arrive at a suitable genetic explanation, the returns-to-heat before 30 days have been separate off from the other embryonic losses and congenital defects. The returns before 30 days might well be explained by poor semen quality of the bull, whereas the other defects could well be caused by a dominant autosomal gene showing incomplete penetrance. The bull Pompon, a normal overlap heterozygote, would have transmitted the gene to all the heterozygotes in his progeny, and about $90 \%$ of these have manifested the defect, either by disappearing prematurely during gestation, or else by showing at birth the anatomical and clinical symptoms described above. It is uncertain whether the bad quality of the sperm is due to the heterozygosity of the bull.

\section{RÉFÉRENCES BIBLIOGRAPHIQUES}

I,AUVERGNe J.-J., I968. Catalogue des anomalies héréditaires des bovins (Bos taurus L.). Bull. tech. Dép. Génét. anim. (Inst. nation. Rech. agron., Fr.), no $\mathrm{I}, 9 \mathrm{I}$ p.

I.AUVERGNe J.-J., Vissac B., I963. Genetical study of " tête de mouton " cattle. XI Int. conf. Genet., 1, 264-265. 